\title{
Novel Approaches to Calculate Nuclear Matrix Elements for Dou- ble Beta Decays
}

\author{
Mihai Horoi ${ }^{1, a}$ \\ ${ }^{1}$ Department of Physics, Central Michigan University, Mount Pleasant, Michigan 48859, USA
}

\begin{abstract}
Neutrinoless double beta decay is a unique process that could reveal physics beyond the Standard Model of particle physics. If observed, it would prove that neutrinos are Majorana particles, and it could give information regarding the neutrino masses and their hierarchy, provided that reliable nuclear matrix elements (NME) can be obtained. The two-neutrino double beta decay is an associate process that is allowed by the Standard Model and it was observed for about ten nuclei. The NME associated with this decay mode could be even more difficult to calculate, but they can be directly related to the experimental half-lives, and they can be constrained using data from charge-exchange reactions. Here we offer a brief overview of the theoretical challenges associated with these two processes, emphasizing the tools necessary to reliably calculate the associated nuclear matrix elements. We also emphasize the role of the competing mechanisms that could contribute to the neutrinoless double beta decay half-life.
\end{abstract}

Neutrinoless double beta decay is a unique process that could reveal physics beyond the Standard Model of particle physics [1, 2]. If observed, it would prove that neutrinos are Majorana particles, and it could give information regarding the neutrino masses and their hierarchy, provided that reliable nuclear matrix elements (NME) can be obtained [1,3-6]. The two-neutrino double beta decay is an associate process that is allowed by the Standard Model and it was observed for about ten nuclei. The NME associated with this decay mode could be even more difficult to calculate [1, 3, 6-10], but they can be directly related to the experimental half-lives, and they can be constrained using data from charge-exchange reactions.

The present contribution offers a brief overview of the theoretical challenges associated with these two processes, emphasizing the tools necessary to reliably calculate the associated nuclear matrix elements. It also emphasizes the role of the competing mechanisms that could contribute to the neutrinoless double beta decay half-life [1,3]. This analysis is very timely given the recent GERDA phase I results [11], which strongly rule out the Klapdor's claim of a positive observation of neutrinoless double beta decay of ${ }^{76} \mathrm{Ge}$ more than a decade ago [12]. In particular, the special case of ${ }^{136} \mathrm{Xe}$ is analyzed. Recent experimental results for its two-neutrino double beta decay mode provide a rather small NME $[13,14]$. We propose a novel shell model approach that can accurately described this small NME without artificially decreasing the Gamow-Teller quenching factor. Having tuned the wave functions to described the two-neutrino double beta half-life, we used them to calculate the neutrinoless NME. Using the recent experimental upper limit of the associated neutrinoless half-life [6] we derive lower limits for the neutrino physics parameters, and in particular, assuming that the domi-

\footnotetext{
ae-mail: mihai.horoi@cmich.edu
} 
nant mechanism contributing to the decay is the exchange of light left-handed Majorana neutrino, we provide lower limits for the effective neutrino mass [3, 4].

For the $2 v \beta \beta$ decay mode the relevant NME is of Gamow-Teller type, $M_{G T}^{2 v}$, and it is shown in Eq. (1) of Ref. [3]. The $2 v \beta \beta$ decay half-life expression is given by

$$
\left[T_{1 / 2}^{2 v, J}\right]^{-1}=G_{J}^{2 v}\left|M_{G T}^{2 v}(J)\right|^{2},
$$

where $G_{J}^{2 v}$ are $2 v \beta \beta$ phase space factors. Specific values of $G_{J}^{2 v}$ for different $2 v \beta \beta$ decay cases can be found in different reviews: for a recent analysis of $G_{J}^{2 v}$ see Ref. [15].

The $2 v \beta \beta$ NME for the decays of ${ }^{48} \mathrm{Ca}$ are given in Table I of Ref. [3]. The shell model calculations were done in the $p f$ model space using a quenching factors 0.74 for the Gamow-Teller operator. One should mention that in the $p f$ model space all spin-orbit partner orbitals are present, and the Ikeda sum-rule is satisfied, thus the full Gamow-Teller strength is accounted. The shell model calculations for ${ }^{136} \mathrm{Xe}$ are usually done in the $j j 55$ model space composed of the $0 g_{7 / 2} 1 d_{5 / 2} 1 d_{3 / 2} 2 s_{1 / 2} 0 h_{11 / 2}$ orbitals. These calculations requires an artificially small quenching factor [16] to describe the small matrix element extracted from recent experimental data $[13,14]$. We used the larger model space $j j 77$ obtained by including the spin-orbit partners $0 g_{9 / 2} 0 h_{9 / 2}$, and under a series of truncations we were able to satisfy the Ikeda sum-rule and to describe the small NME using the standard quenching factor [4].

The $0 v \beta \beta$ decay, $(Z, A) \rightarrow(Z+2, A)+2 e^{-}$, requires the neutrino to be a massive Majorana fermion, i.e. it is identical to the antineutrino [17]. We already know from the neutrino oscillation experiments that some of the neutrinos participating in the weak interaction have mass, and that the mass eigenstates are mixed by the PMNS matrix $U_{l k}$, where $l$ is the lepton flavor and $k$ is the mass eigenstate number (see e.g. Ref. [1]). However, the neutrino oscillations experiments cannot decide the mass hierarchy, the mass of the lightest neutrino, and some of the CP non-conserving phases of the PMNS matrix (assuming that neutrinos are Majorana particles). Considering only contributions from the exchange of light, left-handed(chirality), Majorana neutrinos [1, 2], the $0 v \beta \beta$ decay half-live is given by

$$
\left[T_{1 / 2}^{0 v}\right]^{-1}=G^{0 v}\left|M_{v}^{0 v}\right|^{2}\left(\frac{\left|\left\langle m_{\beta \beta}\right\rangle\right|}{m_{e}}\right)^{2}
$$

Here, $G^{0 v}$ is the phase space factor, which depends on the $0 v \beta \beta$ decay energy, $Q_{\beta \beta}$, the charge of the decaying nucleus $Z$, and the nuclear radius [15]. The effective neutrino mass, $\left\langle m_{\beta \beta}\right\rangle$, is related to the neutrino mass eigenstates, $m_{k}$, via the left-handed lepton mixing matrix, $U_{e k}$,

$$
\left\langle m_{\beta \beta}\right\rangle / m_{e} \equiv \eta_{v L}=\sum_{k=l i g h t} m_{k} U_{e k}^{2} / m_{e}
$$

$m_{e}$ is the electron mass $[1,3,5]$. Assuming that one can unambiguously measure a $0 v \beta \beta$ half-life, and one can reliably calculate the NME for that nucleus, one could use Eqs. (2) and (3) to extract information about the lightest neutrino mass and the neutrino mass hierarchy [1]. In addition, one could consider the contribution from the right-handed currents to the effective Hamiltonian, which can mix light and heavy neutrinos of both chiralities (L/R)

$$
v_{e L}=\sum_{k=\text { light }} U_{e k} v_{k L}+\sum_{k=\text { heavy }} U_{e k} N_{k L}, \quad v_{e R}=\sum_{k=\text { light }} V_{e k} v_{k R}+\sum_{k=h e a v y} V_{e k} N_{k R}
$$


where $N_{k}$ are the heavy neutrinos that are predicted by several see-saw mechanisms for neutrino masses [1]. $U_{l k}$ and $V_{l k}$ are the left and right-handed components of the unitary matrix that diagonalizes the neutrino mass matrix $[1,2]$. One should also mention that there are several other mechanisms that could contribute to the $0 v \beta \beta$ decay, such as the exchange of supersymmetric (SUSY) particles (e.g. gluino and squark exchange [1]), etc, whose effects are not directly related to the neutrino masses, but indirectly via the Schechter-Valle theorem [17]. Assuming that the masses of the light neutrinos are smaller than $1 \mathrm{MeV}$ and the masses of the heavy neutrinos, $M_{k}$, are larger than $1 \mathrm{GeV}$, the particle physics and nuclear structure parts get separated, and the inverse half-life can be written as

$$
\left[T_{1 / 2}^{0 v}\right]^{-1}=G^{0 v}\left|\eta_{v L} M_{v}^{0 v}+<\lambda>\tilde{X}_{\lambda}+<\eta>\tilde{X}_{\eta}+\left(\eta_{N L}+\eta_{N R}\right) M_{N}^{0 v}+\eta_{\lambda^{\prime}} M_{\lambda^{\prime}}^{0 v}+\eta_{\tilde{q}} M_{\tilde{q}}^{0 v}\right|^{2},
$$

where $\eta_{v L}$ was defined in Eq. (3), and

$$
\begin{aligned}
\eta_{N L} & =\sum_{k=\text { heavy }} U_{e k}^{2} \frac{m_{p}}{M_{k}}, \quad \eta_{N R} \approx\left(\frac{M_{W_{L}}}{M_{W_{R}}}\right)^{4} \sum_{k=\text { heavy }} V_{e k}^{2} \frac{m_{p}}{M_{k}}, \\
<\lambda> & =\epsilon \sum_{k=\text { light }} U_{e k} V_{e k}, \quad<\eta>=\left(\frac{M_{W L}}{M_{W R}}\right)^{2} \sum_{k=\text { light }} U_{e k} V_{e k} .
\end{aligned}
$$

Here $\epsilon$ is the mixing parameter for the right heavy boson $W_{R}$ and the standard left-handed heavy boson $W_{L}, W_{R} \approx \epsilon W_{1}+W_{2}, M_{W R}$ and $M_{W L}$ are their respective masses, and $m_{p}$ is the proton mass. The $\eta_{\lambda^{\prime}}$ and $\eta_{\tilde{q}}$ are the R-parity violation contributions in supersymmetric (SUSY) Grand Unified Theories (GUT) related to the long range gluino exchange and squark-neutrino mechanism, respectively [1].

Constraints from colliders experiments suggest that terms proportional with the mixing angles, $\epsilon$, $U_{e k(h e a v y)}$, and $V_{e k(l i g h t)}$ are very small [18]. The present limits are $|<\lambda>|<10^{-8}$ and $|<\eta>|<10^{-9}$, but they are expected to be smaller. In addition, the contributions from $\tilde{X}_{\lambda}$ and $\tilde{X}_{\eta}$ terms in Eq. (5) would produce angular and energy distribution of the outgoing electrons different than that coming from all other terms [19], and these signals are under investigation at SuperNEMO [19]. Here we assume that these contributions are small and can be neglected. In addition, if $\langle\lambda\rangle$ is small, Eq. (6) suggests that $\eta_{N L}$ is small. Then, the half-life can be written as (here $\eta_{N}=\eta_{N R}$ )

$$
\left[T_{1 / 2}^{0 v}\right]^{-1}=G^{0 v}\left|\eta_{v L} M_{v}^{0 v}+\eta_{N} M_{N}^{0 v}+\eta_{\lambda^{\prime}} M_{\lambda^{\prime}}^{0 v}+\eta_{\tilde{q}} M_{\tilde{q}}^{0 v}\right|^{2}
$$

The structure of $M_{j}^{0 v}, j=\left(v, N, \lambda^{\prime}, \tilde{q}\right)$, is the same as that described in Eqs. (5)-(8) of Ref. [3], with the neutrino potentials $H_{\alpha}(r)$ described in Refs. [3,5]) (see also page 68 of Ref. [1]). A detailed description of the matrix elements for the $j j$-coupling scheme consistent with the conventions used by modern shell model effective interactions is given in Ref. [5]. We included in the calculations $[3,5]$ the recently proposed higher order terms in the nucleon currents, three parametrization of the short-range correlations (SRC) effects proposed in the literature [5], finite size (FS) effects, the effects of the average energy of the states in the intermediate nucleus, and we treated carefully few other parameters entering into the calculations.

The results for all NME entering Eq. (7) for the transition of ${ }^{48} \mathrm{Ca}$ to the $0_{1}^{+}$g.s. and first excited $0_{2}^{+}$ state of ${ }^{48} \mathrm{Ti}$ are presented in Table II of Ref. [3] for two sets of short-range correlations (SRC). Table II of Ref. [4] presents similar matrix elements for the decay of ${ }^{136} \mathrm{Xe}$. Based on these calculations and using the experimental lower limit of the half-life, one can extract the "single-mechanism dominance" 
upper limits for $\left|\eta_{j}\right|$, where $j=(v L), N, \lambda^{\prime}, \tilde{q}$. Using the phase-space factor from Ref. [15], we obtained the upper limits for $\left|\eta_{j}\right|$ shown in Table III of Ref. [3] $\left({ }^{48} \mathrm{Ca}\right)$ and Table II of Ref. [4] $\left({ }^{136} \mathrm{Xe}\right)$. Alternatively, assuming that two or more mechanisms contributing to the half-life in Eq. (7) compete, one could use the experimental data from several isotopes to assess the contribution of each mechanism [20]. Clearly, this scenario requires as many as possible accurate half-lives and associated NMEs. If the exchange of light neutrino will be determined as the dominant mechanism, then our results could possible be used to decide the light neutrino mass hierarchy and the lowest neutrino mass [1].

In conclusion, we analyzed the $2 v \beta \beta$ and several mechanisms that could contribute to the $0 v \beta \beta$ decays of ${ }^{48} \mathrm{Ca}$ and ${ }^{136} \mathrm{Xe}$ using shell model techniques. We described very efficient techniques to calculate accurate $2 v \beta \beta \mathrm{NME}$ for cases that involve large shell model dimensions. These techniques were tested for the case of ${ }^{48} \mathrm{Ca}$, and we provided NME and half-lives for $2 v \beta \beta$ transitions to the g.s. and excited states of ${ }^{48} \mathrm{Ti}$. We have also shown that by including in model space $j j 55$ all spin-orbit partners one can satisfy the Ikeda sum-rule and describe the small $2 v \beta \beta$ matrix element for ${ }^{136} \mathrm{Xe}$ without recourse to artificially small quenching factors. We also reviewed the main contributing mechanisms to the $0 v \beta \beta$ decay, and we showed that based on the present constraints from colliders one could reduce the contribution to the $0 v \beta \beta$ half-life to the relevant terms described in Eq. (7). We extended our recent analysis [5] of the $0 v \beta \beta$ NME for ${ }^{48} \mathrm{Ca}$ to include the heavy neutrino exchange NME, the long range gluino exchange NME, and the squark-neutrino mechanism NME. Shell model results of these new NME for the $0 v \beta \beta$ transitions to low-lying $0^{+}$states in ${ }^{48} \mathrm{Ti}$ and ${ }^{136} \mathrm{Ba}$ were also presented.

U.S. NSF grant no. PHY-1068217 and DOE grant no. DE-SC0008529 are acknowledged.

\section{References}

[1] J.D. Vergados, H. Ejiri, and F. Simkovic, Rep. Prog. Phys. 75, 106301 (2012).

[2] T. Tomoda, Rep. Prog. Phys. 54, 53 (1991).

[3] M. Horoi, Phys. Rev. C 87, 014320 (2013).

[4] M. Horoi and B.A. Brown, Phys. Rev. Lett. 110, 222502 (2013).

[5] M. Horoi and S. Stoica, Phys. Rev. C 81, 024321 (2010).

[6] M. Horoi, S. Stoica, B.A. Brown, Phys. Rev. C 75, 034303 (2007).

[7] M. Horoi, J. Phys. Conf. Series 413, 012020 (2013).

[8] M. Horoi, AIP Conference Proceedings 1498, 97 (2012).

[9] M. Horoi, AIP Conference Proceedings 1417, 157 (2011).

[10] M. Horoi, AIP Conference Proceedings 1304, 106 (2010).

[11] M. Agostini et al., arXiv:1307.4720 [nucl-ex] (2013).

[12] V. Klapdor-Kleingrothaus et al., Phys. Lett. B 586, 198 (2004).

[13] M. Auger et al., Phys. Rev. Lett. 109, 032505 (2012).

[14] A. Gando et al., Phys. Rev. Lett. 110, 062502 (2013).

[15] J. Kotila and F. Iachello, Phys. Rev. C 85, 034316 (2012).

[16] E. Caurier, F. Nowacki, and A. Poves, Phys. Lett. B 711, 62 (2012).

[17] J. Schechter and J.W.F. Valle, Phys. Rev. D 25, 2951 (1982).

[18] S. P. Das, F. F. Deppisch, O. Kittel, and J. W. F. Valle, Phys. Rev. D 86, 055006 (2012).

[19] R. Arnold et al., arXiv:1005.1241 [hep-ex] (2010).

[20] A. Faessler, A. Meroni, S.T. Petcov, F. Simkovic, and J. Vergados, Phys. Rev. D 83, 113003 (2011). 